\title{
Aplicação do Sistema de Inferência Nebuloso na Determinação de Medicina Preventiva no Sistema de Saúde Complementar
}

\author{
Igor Barone de Medeiros, Maria Augusta Soares Machado \\ Faculdades IBMEC - Brasil \\ mmachado@ibmecrj.br
}

\begin{abstract}
Resumo
A aplicação do Sistema de Inferência Nebuloso foi realizada na tentativa de direcionar os custos médicos de operadoras de planos de saúde mediante o estudo de variáveis, previamente, selecionadas por grupos médicos de atuação no mercado. Esta medida irá influenciar as decisões pós entrevista qualificada através do mapeamento do risco dos novos clientes.

Este estudo tangibiliza a atual situação do mercado de saúde privada no Brasil, tendo como premissa os escassos recursos da Saúde Pública. Em consequiência há a tentativa do governo em atender a todos de maneira igualitária, contudo os benefícios a serem oferecidos são, infelizmente, segmentados por não haver recursos para toda a população.

O PIB no Brasil eqüivale a $60 \%$ do investimento norte americano em saúde, fonte do relatório do Banco Mundial de 1993, o que representa uma longa jornada de trabalho de nossa economia para o aumento da viabilidade de sobrevivência neste mercado que esta cada vez mais acirrada devido a competição que se encontra o setor de saúde complementar. O objetivo central é restaurar a capacidade comercial deste mercado engessado.

A organização de pensamentos dos administradores de saúde (muitos são médicos) ainda está ao redor dos procedimentos médicos, pois são eles que alimentam economicamente os Hospitais. Seguindo este foco, são apresentadas vertentes que caracterizam a prevenção como a maior fomentadora de recursos para ambos, operadoras e hospitais, tendo em vista a distribuição dos custos médicos e conseqüentemente o aumento da capacidade dos hospitais em absorver novas técnicas de tratamento, garantindo maior qualidade de vida para os clientes, além de abrir portas para novas parcerias.
\end{abstract}

Palavras chave: matemática nebulosa, sistema de inferência nebuloso,medicina preventiva

\begin{abstract}
The application of Fuzzy Inference System was carried out in attempt to focuses medical costs of operators of heath's plans, by means of previous studies of variables, selected by acting medical groups. This measure will influence the decisions powders glimpsed qualified through mapping the risk of the new clients.

This study illustrates the current situation of prived health 's market in Brazil, taking as a premise the scarce resources of the Public Health. In consequence, there is the attempt of the government in attending to all in egalitarian way, nevertheless, the benefits to be offered are, unfortunately, segmented, since there are no resources for the whole population.

Internal Gross Product in Brazil is equal to $60 \%$ of the northern American investment in health, based on 1993 BIRD 's report, which represents a long working day of our economy to increase the viability of survival in this market.The central objective is to restore the commercial capacity of this put in plaster market.

The organization of thoughts of the administrators of health (many people are doctors) is still around the medical proceedings, based on the fact that they feed economically the Hospitals. Following this focus, this paper presents tendencies that characterizes the prevention like the biggest encouraging one of resources for both, operators and hospitals, having in mind the distribution of the medical costs and consequently the increase of the capacity of the hospitals in absorbing new techniques of treatment, guaranteeing bigger quality of life for the clients, besides opening doors for new partnerships.
\end{abstract}

Key words: fuzzy logic, fuzzy inference system, preventive medicine 


\section{Introdução}

A situação do setor de saúde suplementar abre portas para medidas inovadoras, onde novas idéias de trabalho devem ser testadas e implantadas, proporcionando uma taxa de crescimento muito significativa para pessoas com espírito empreendedor, além de ser um setor onde há uma grande movimentação financeira.

O sistema de saúde brasileiro está em profunda transição, onde diversos pontos de sua gestão estão sendo questionados, por exemplo podemos citar: a administração da Agência Nacional de Saúde Suplementar e a inconsistência da lei 9656/1998, além da falta de investimento em capacidade intelectual. Esse momento de transição está sendo crucial para as operadoras de planos de saúde que podem fechar as suas portas a qualquer momento caso seja aplicado as devidas medidas da Agencia Nacional de Saúde, exemplo, empresas com patrimônio líquido negativo [2]. Não obstante, recursos para o setor encontram-se cada vez mais escassos devido ao crescimento acelerado dos custos médicos e de diagnóstico, maiores que a inflação.

Sendo assim é de total aplicação o estudo usando inferência nebulosa (Lógica Fuzzy) para aferir os riscos de uma carteira. Não podemos deixar de considerar que o sistema de saúde pública se encontra privilegiada, já que é reduzido o número de incidentes em sua rede hospitalar devido existência do mercado privado que se não bastasse e cobrado o ressarcimento ao SUS quando credenciados são atendimento na rede pública por alguma eventualidade.

\section{Objetivo}

O estudo deste tema possui como objetivo estimular a tomada de novas decisões dentro do setor de saúde, ou seja fomentar a criação de novas estratégias que consigam tornar sustentável a administração das operadoras, hospitais e todos os envolvidos no negócio saúde.

As informações necessárias para as devidas decisões nunca são encontradas pela Agência Nacional de Saúde [2] , a qual taxa as operadoras sobre cada beneficiário o que leva a sonegação de informações por parte das atingidas, caracterizada na diferença da base de dados informada pela Abramge e pela ANS, tornando assim escassas análises precisas do setor.

Tendo em vista toda a insatisfação deste mercado, movido por um sistema administrativo carente e por princípios de interesse dos próprios dirigentes, esse trabalho possui o interesse de expor uma medida de identificação de problemas médicos de beneficiários através de um sistema nebuloso que irá funcionar como uma inteligência médica artificial, em larga escala devido à manipulação de banco de dados, aumentando assim a capacidade das operadoras e prestadores em absorver novos beneficiários e controlar melhor os seus custos.

\section{O Setor de Saúde Pública e Privada Mundial e Nacional}

\subsection{Análise Macro Econômica do Setor de Saúde Pública}

Os dados apresentados nesta análise são fonte de dados do relatório do Banco Mundial de 1993.

A administração de organizações de saúde, incluindo as operadoras de planos de assistência suplementar à saúde, é uma das mais complexas atividades no mundo dos negócios da atualidade. O mercado global de saúde realiza aplicações de 1,7 trilhões de dólares, de acordo com dados do Banco Mundial de 1993.

Nos últimos 40 anos, a expectativa de vida aumentou mais do que em toda a história pregressa da humanidade. Em 1950, a expectativa de vida nos países em desenvolvimento era de 40 anos; em 1990, já subira para 63 anos. Em 1950 em cada 100 crianças, 28 morriam antes de completar cinco anos; em 1990, esse número já caíra a 10. A varíola, que matava anualmente mais de 5 milhões de pessoas no início dos anos 50, foi totalmente erradicada. As vacinas reduziram sensivelmente os casos de sarampo e de pólio. Todos esses progressos não traduzem apenas ganhos expressivos e diretos de bem estar, também diminuem o ônus econômico imposto por trabalhadores com saúde precária e de estudantes doentes ou faltosos. Esses progressos se devem em parte ao aumento das rendas e à melhoria da educação em todo o mundo e, em parte, aos esforços dos governos para expandir os serviços de saúde, que também se beneficiaram dos avanços tecnológicos.

\begin{tabular}{|c|c|c|c|c|c|}
\hline $\begin{array}{c}\text { Região } \\
\text { Demográfica }\end{array}$ & $\begin{array}{l}\text { População } \\
\text { (em } \\
\text { milhões) }\end{array}$ & $\begin{array}{c}\% \\
\text { população } \\
\text { global }\end{array}$ & $\begin{array}{c}\text { Gastos c/ } \\
\text { saúde } \\
\text { (bilhões de } \\
\text { US\$) }\end{array}$ & $\begin{array}{c}\% \text { dos } \\
\text { gastos } \\
\text { globais c/ } \\
\text { saúde (US\$) }\end{array}$ & $\begin{array}{c}\text { Gastos per } \\
\text { capita c/ } \\
\text { saúde (US\$) }\end{array}$ \\
\hline $\bar{A}$ Arica & 510 & 10 & 12 & 1 & 24 \\
\hline Índia & 850 & 16 & 18 & 1 & 21 \\
\hline China & 1134 & 22 & 13 & 1 & 11 \\
\hline Ásia e Ilhas & 683 & 13 & 42 & 2 & 61 \\
\hline $\begin{array}{l}\text { América } \\
\text { Latina e } \\
\text { Caribe }\end{array}$ & 444 & 8 & 47 & 3 & 105 \\
\hline $\begin{array}{l}\text { Oriente } \\
\text { Médio } \\
\text { Crescente } \\
\end{array}$ & 503 & 10 & 39 & 2 & 77 \\
\hline $\begin{array}{l}\text { Antigas } \\
\text { economias } \\
\text { socialistas } \\
\text { européias }\end{array}$ & 346 & 7 & 49 & 3 & 142 \\
\hline $\begin{array}{l}\text { Economia de } \\
\text { mercados } \\
\text { consolidados }\end{array}$ & 798 & 15 & 1483 & 87 & 1860 \\
\hline Mundo & 5268 & 100 & 1703 & 100 & 329 \\
\hline
\end{tabular}

Tabela 1- Gastos Globais com Saúde

\subsection{Problemas no setor:}

- Má alocação de recursos: Na área de saúde, o dinheiro público é gasto com intervenções de pouca eficácia em função dos custos, como 
cirurgias para extirpar os mais variados tipos de câncer, enquanto continua carente de recursos e intervenções cruciais de grande eficácia em termos de custo, como o tratamento da tuberculose e de doenças sexualmente transmissíveis (DST). Em certos países, um único hospital-escola pode absorver $20 \%$ ou mais do orçamento do Ministério da Saúde, muito embora as instalações mais adequadas para quase todas as intervenções eficazes em termos de custo sejam as de nível mais elementar.

- Desigualdade social: Os pobres não têm acesso a serviços de saúde e quando conseguem recebem atendimento de má qualidade. Os gastos públicos com saúde privilegiam os mais favorecidos, sob a forma de assistência médica gratuita ou abaixo do custo em hospitais públicos sofisticados de atendimento terciário (para patologias de difícil tratamento), e de subsídios ao seguro-saúde público e privado.

- Ineficiência: Grande parte do dinheiro gasto com saúde é desperdiçada; compram-se produtos farmacêuticos de marca registrada ao invés de medicamentos genéricos, distribuem-se e supervisionam-se mal os que trabalham na área, e subutilizam-se leitos hospitalares.

- Custos elevados: Em alguns países em desenvolvimento de renda média, as despesas com assistência médica crescem muito mais depressa que a renda. $\mathrm{O}$ aumento do número de clínicos gerais e especialistas, a disponibilidade de novas tecnologias médicas e a expansão do seguro-saúde gera um rápido aumento da demanda de exames, procedimentos e tratamentos dispendiosos.

\subsection{Análise Macro Econômica do Setor de Saúde Privada}

As fontes dos dados apresentados são da Agencia Nacional de Saúde e Abramge (Associação Brasileira de Medicina de Grupo).

As várias modalidades de assistência médica suplementar combinam a função de seguro com a de prestação de serviços de atenção médica. E incluem, como principais formas institucionais, a medicina de grupo, as cooperativa médicas, os planos próprios das empresas (autogestões e planos administrados) e os seguros de saúde.

O âmbito da assistência médica suplementar compreende empresas financeiras e não financeiras a atuarem, direta e indiretamente, na área de prestação de serviços médicos, serviços hospitalares e serviços auxiliares de diagnóstico e terapêutica, à população; os serviços prestados por estas entidades são unicamente de natureza mercantil, isto é, a prestação dos serviços gera, necessariamente, uma receita [4,5].

Já as despesas com esses serviços de assistência médica envolvem diversos tipos de gastos. E para bom entendimento deve-se acompanhar o seguinte raciocínio, em primeiro lugar, os pagamentos efetuados pela população aos planos e seguros de saúde, que podem compreender planos de pré-pagamentos e póspagamento.

Em segundo lugar, os pagamentos realizados pelas empresas (financeiras e não financeiras) gestoras de planos e seguros de saúde às empresas, contratadas (ou conveniadas) para a prestação de serviços de natureza médica, hospitalar e de serviços diagnósticoterapêuticas à população usuária do sistema. Em terceiro, as despesas efetuadas pelas empresas (não financeiras) da área médica com a compra do material (insumos e equipamentos hospitalares) necessário ao exercício da assistência aos seus usuários [7].

O sub-setor de assistência médica suplementar, portanto, compreende um amplo, diversificado e, muitas vezes, gerando conflitos de interesses de vários setores (empresas financeiras e não financeiras, governo e família).

É possível dizer que as receitas das empresas de planos de saúde são predominantemente compostas pela venda de planos e seguros de saúde às famílias. Esses planos podem ser adquiridos diretamente pelas mesmas (planos individuais ou familiares) ou pelos empregadores dos membros destas famílias (planos empresarias ou coletivos). Quando os empregadores são responsáveis pelo plano de saúde, é considerado que houve fluxo transferências de renda para as famílias de valor equivalente ao que deixam de desembolsar com a contratação de planos individuais ou familiares de assistência médica suplementar $[9,10]$.

Depreende-se assim que as famílias são as únicas beneficiadas finais, diretas, dos serviços de atenção médica, independentemente da forma como se realizam os fluxos monetários entre famílias, empresas empregadoras, empresas de planos de saúde, empresas prestadoras de assistência médica e governo.

\section{Suposições}

Mediante a avaliação dos aspectos sócio-culturais, econômicos e financeiros apresentados a aplicação da Lógica Nebulosa na gestão dos serviços de saúde é uma abordagem recente e imprescindível na busca de uma eficiente e efetiva prestação de cuidados. Os conceitos de prevenção de doenças e promoção de saúde sugerem meios de elevar o nível físico e mental dos indivíduos e de expandir o uso dos serviços. As instituições devem criar novas dimensões na oferta de serviços, buscando responder a desafios da promoção da saúde e da prevenção. Sugere-se que o conceito de elevar o nível de saúde seja modificado da perspectiva orientada para doença, para a perspectiva orientada para a saúde.

Para que as nações melhorem o nível de saúde de suas populações, é fundamental que reorganizem suas prioridades relacionadas aos cuidados de saúde, dando maior ênfase a prevenção das doenças e a promoção da saúde. É necessário que os serviços compreendam os problemas de saúde de seus pacientes e estendam a comunidade e as populações [11,13] . 
O principio de Integralidade dos serviços de saúde preconizado pela nossa constituição, deve ser aplicado inclusive no sistema suplementar e suas ações devem ser integradas desde os serviços de atenção primaria, preferencialmente a porta de entrada para aqueles que apresentem agravos ou problemas de saúde de qualquer espécie. Os serviços de saúde precisam estender suas ações à comunidade e não só atender os seus pacientes que os procuram. Tais ações devem ser voltadas a grupos em situações de risco ambiental, estilo de vida, ou ainda, de hereditariedade. Os prestadores de serviços devem ter um compromisso com a comunidade como um todo e com seus membros individualmente, desenvolvendo, inclusive programas de prevenção às doenças.

Os custos da assistência à saúde têm aumentado, enquanto as taxas de ocupação hospitalar têm diminuindo. Paralelamente, as taxas de sobrevivência as doenças crônicas e a expectativa de vida têm aumentado, o que aponta para a necessidade de se investir em prevenção secundária e terciária. A prevenção primaria apresenta, para os prestadores de serviços de saúde, a oportunidade de lidar com estas mudanças.

A instituição de saúde e o médico não devem arcar com toda a carga para a melhora da prática de prevenção de doenças e promoção à saúde, no entanto essas práticas necessitam ser aplicadas para que os indivíduos e a sociedade, de um modo geral, se tornem progressivamente motivados para assumir uma maior responsabilidade sobre sua saúde [18,19].

\subsection{Prevenção de doenças e agravos e promoção da saúde}

Tradicionalmente divide-se a prevenção em três níveis gerais. São eles:

- Primária, onde há interrupção do desenvolvimento de uma doença antes que ela ocorra;

- Secundária, onde ocorre o diagnostico precoce e tratamento da doença;

- Terciária, onde são realizadas a reabilitação ou recuperação das funções normais.

A prevenção primaria consiste na adoção de medidas apropriadas a manutenção da saúde geral e de elementos protetores específicos, como imunização, saneamento ambiental e proteção contra acidentes e riscos ocupacionais, que são muito eficazes na redução da mortalidade e morbidez de muitos agravos e doença [3]. As medidas gerais de promoção de saúde incluem aquelas que interfiram no estilo de vida, nos fatores ambientais e biológicos e, certamente, podem proporcionar uma maior redução nos indicadores de mortalidade. Esse nível de intervenção, não importa em qual elemento do Campo de Saúde, deve promover estratégias para difusão da saúde e prevenção de doenças.

O diagnostico precoce e o tratamento de doenças faz parte da prevenção secundária. O diagnóstico precoce do câncer, de hipertensão, diabetes, doenças sexualmente transmissíveis e outras doenças passíveis de tratamento e cura é o seu objetivo. No caso de algumas doenças crônicas degenerativas, e ligadas ao envelhecimento, a prevenção secundaria é importante na limitação da incapacidade, prevenindo complicações ou seqüelas.

A prevenção terciária é responsável em evitar total incapacidade do paciente no caso de uma doença já instalada e que tenha promovido seqüelas, no momento em que as alterações anatômicas e fisiológicas se manifestem, com intensidade variável. O seu objetivo é recuperar o indivíduo, dando-lhe o maior grau possível de auto-suficiência [14].

\section{Delimitação do estudo}

O estudo delimita-se em abordar o mecanismo de Inferência Nebulosa para aplicação na gestão das operadoras de plano de saúde. A ferramenta utilizada foi o Matlab. A partir da formação de um conjunto de variáveis será explicada a importância do apoio administrativo na aplicação de questionários em entrevistas qualificadas de carteiras de planos de saúde, com o objetivo de obter cada vez mais informações sobre os usuários resultando em um controle e planejamento mais efetivo através do agrupamento dos entrevistados em diferentes grupos de risco. Isso implicará no estudo de sinistralidade através da Prevenção de doenças e agravos para promoção da saúde.

\section{Relevância do estudo}

A saúde é um dos bens sociais mais preocupantes em todo o mundo. Sua atribuição é de caracter importantíssimo para o desenvolvimento de uma sociedade. Sempre será algo questionado pela população quando não houver por parte dos países responsabilidade em relação à administração da saúde pública. Sendo assim, cabe à administração da saúde Privada continuar inovando e correspondendo as necessidades da população. Não obstante, é possível alavancar o retorno desejado pelo investimento em planejamento, controle e avaliação da base de dados formada através Lógica Nebulosa.

\section{Referencial teórico}

\subsection{Inteligência Artificial}

\subsubsection{Histórico da Inteligência Artificial}

A Inteligência Artificial (IA) não é recente. Sua história se inicia nos idos dos anos 40, onde havia alguma pesquisa em torno de sequiências de estratégia e análise do funcionamento do cérebro com objetivos de formalização de seu comportamento. Estes dois ramos de pesquisa são dissociados entre si, sem nenhuma preocupação com a construção de uma Inteligência 
Artificial. Buscavam-se, apenas, novas alternativas de utilização do computador, ainda em projeto [12].

Mas, com o passar dos anos, foram sendo distinguidas duas linhas de pesquisa: uma biológica, calcada em torno do funcionamento do cérebro e dos neurônios; e outra, fruto do estudo da cognição, do raciocínio.

\begin{abstract}
Anos 70
Para a linha biológica, essa foi uma década negra. Apesar disso, houve pesquisadores que, por outros caminhos, chegaram a novas concepções de redes neurais artificiais. Estas concepções analisavam o aprendizado de informações como sendo fruto de uma união das potencialidades de redes de neurônios interagindo entre si. Nasciam as redes neurais representadas na forma de mapas cerebrais, onde não havia o aprendizado de um neurônio, mas de toda uma rede, através do compartilhamento de recursos. Já na linha psicológica, estudos mais aprofundados demonstraram o óbvio: que não seria possível à representação numa máquina dos estados mentais humanos responsáveis pelo pensamento. A saída para esta linha de desenvolvimento era dada por uma empresa: a Rand Corporation. Foi de sua equipe de pesquisa que partiram os sistemas especialistas, os quais foram responsáveis pela ampliação da Inteligência Artificial Tradicional, usando manipulação simbólica.
\end{abstract}

\section{Anos 80}

As redes neurais artificiais tiveram seu reconhecimento recuperado através do físico John Hopfield, que em 1982 provou ser possível à simulação de um sistema físico através de um modelo matemático baseado na teoria das redes neurais.

Assim, em 1986, uma equipe de especialistas das mais diversas áreas reuniu-se para validar as pesquisas em torno das redes neurais, possibilitando a volta da pesquisa nesta linha. Uma das formas de recuperação do prestígio das redes neurais foi à proposta de um método de treinamento, chamado retropropagação ("backpropagation"), que ampliava o potencial do perceptron de modo a permitir a superação das limitações do modelo primitivo. Enquanto isso, na IA Tradicional, ampliavam-se as técnicas e aplicações dos sistemas especialistas que só mais tarde começaram a usar a abordagem conexionista (com redes neurais). Além disso, houve o interesse de trabalho conjunto com outras áreas, tais como interfaces inteligentes, sistemas de apoio à decisão, controle de robôs, etc.

\section{Anos 90}

Nessa década, as redes neurais tiveram uma explosão exponencial de aplicações e desenvolvimento de modelos. São centenas de propostas de novos ou aperfeiçoamento de modelos a cada ano, tal o interesse pela área. A partir daí, consolidam-se as redes neurais como parte integrante do estudo da Inteligência Artificial propriamente dita. Reconhece-se, também, que os paradigmas biológico e psicológico são complementares e necessários para sistemas mais evoluídos. Desta forma, começam nesta década a serem construídos os chamados Sistemas Híbridos.

Estes sistemas são a uniões das concepções das duas linhas de pesquisa, permitindo a construção de grandes sistemas que pretendem abranger uma forma mais completa de representação do comportamento humano. Ideal este da própria Inteligência Artificial.

\subsubsection{Saber Especializado}

Quais as tarefas que são mais fáceis de serem programadas no computador? Quais são mais complexas? O que é mais simples de formalizar: um jogo ou a visão? A linguagem ou um diagnóstico médico? Para responder a estas perguntas, deve-se investigar os processos subliminares às tarefas realizadas pelo corpo, realizar uma formalização destes processos e implementá-los no computador.

As tarefas que envolvem percepção, por exemplo, são fruto de todo o sistema nervoso atuando como receptor de sinais do meio ambiente. É uma trama complexa de sensores (células nervosas em contato com o ambiente) e memória (reações aos impulsos nervosos que estão armazenados no cérebro). Infelizmente não há um completo domínio de como é feita a recepção e processamento destes sinais. Obviamente, há estudos isolados de mecanismos de visão, voz, olfato etc., porém dependem ainda de muita pesquisa para se chegar a uma formalização consistente destes processos.

Outras tarefas que estão sujeitas a muita pesquisa são as da movimentação. Os maiores problemas para a formalização da motricidade humana são os de aproximação de deslocamento de um membro ou do próprio corpo, e de equilíbrio do corpo. A aproximação de um membro de um objeto exige inúmeros cálculos de física, além de necessitar de outros cálculos que permitam o ajuste preciso em função da direção. Já o equilíbrio de um corpo durante o deslocamento também exige inúmeros cálculos instantâneos para o controle dos membros envolvidos na movimentação. $\mathrm{O}$ reconhecimento de características de uma pessoa, tão facilmente realizada por nós, exige um grande préprocessamento de dados para a depuração das informações mais relevantes, que levem a uma efetiva memorização destas características. É claro que, para memorizar, é necessária a recepção de sinais do meio, através dos canais da percepção. Torna-se necessário, então, vencer a etapa da percepção para chegarmos à classificação efetiva de características das pessoas, permitindo o reconhecimento posterior. Neste caso, além da percepção, são necessários estudos que identifiquem os mecanismos da memória, sejam eles de aprendizado ou de reconhecimento. Há muitos estudos realizados que buscam formalizar os processos mentais, porém são ainda incipientes se comparados ao potencial do ser humano. Sendo assim, não é possível 
uma implementação computacional robusta, capaz de imitar satisfatoriamente a memória humana.

Em contraposição com o senso comum há o saber especializado, o qual possui objetivo e aplicações bem específicos, dentro de um universo delimitado. Tarefas como armar uma estratégia num jogo, realizar cálculos matemáticos ou até mesmo solucionar um problema em nossa profissão não são para nós sempre de simples resolução, o que nos exige um esforço de raciocínio. Paradoxalmente, estas tarefas são mais facilmente implementáveis em computador, uma vez que possuem uma área bem definida de aplicação, possuindo então um contexto de conceitos e regras a serem aplicados para a resolução de um problema específico.

Há um conjunto de tarefas que exige análises de possibilidades para chegar-se a uma solução. Este tipo de tarefas é chamado de formais por terem uma aplicabilidade apenas em áreas fora do mundo real, tais como jogos e problemas matemáticos. No caso de jogos, torna-se necessária a organização das ações e reações dos jogadores, todas elas previsíveis e portanto manipuláveis dentro de regras claras. Nos problemas matemáticos, é semelhante, havendo regras para a construção de formas geométricas, encadeamento de expressões de lógica e derivação no cálculo integral, entre outras aplicações. Estas são, portanto, aplicações situadas dentro de contextos abstratos com variáveis previsíveis.

\subsubsection{Inferência Nebulosa}

Aristóteles, filósofo grego (384 - 322 a.C.), foi o fundador da ciência da lógica, e estabeleceu um conjunto de regras rígidas para que conclusões pudessem ser aceitas como logicamente válidas. O emprego da lógica de Aristóteles levava a uma linha de raciocínio lógico baseado em premissas e conclusões [1]. Como por exemplo: se é observado que "todo ser vivo é mortal" (premissa 1), a seguir é constatado que "Sarah é um ser vivo" (premissa 2), como conclusão tem que "Sarah é mortal". Desde então, a lógica Ocidental, assim chamada, tem sido binária, isto é, uma declaração é falsa ou verdadeira, não podendo ser ao mesmo tempo parcialmente verdadeira e parcialmente falsa. Esta suposição e a lei da não contradição, que coloca que "U e não U" cobre todas as possibilidades, formam a base do pensamento lógico Ocidental.

A Lógica Nebulosa (Fuzzy Logic) viola estas suposições. O conceito de dualidade, estabelecendo que algo pode e deve coexistir com o seu oposto, faz a lógica nebulosa parecer natural, até mesmo inevitável. A lógica de Aristóteles trata com valores "verdade" das afirmações, classificando-as como verdadeiras ou falsas. Não obstante, muitas das experiências humanas não podem ser classificadas simplesmente como verdadeiras ou falsas, sim ou não, branco ou preto. Por exemplo, é aquele homem alto ou baixo? A taxa de risco para aquele empreendimento é grande ou pequena? Um sim ou um não como resposta a estas questões é, na maioria das vezes, incompleta. $\mathrm{Na}$ verdade, entre a certeza de ser e a certeza de não ser, existem infinitos graus de incerteza. Esta imperfeição intrínseca à informação representada numa linguagem natural, tem sido tratada matematicamente no passado com o uso da teoria das probabilidades.

Contudo, a Lógica Nebulosa, com base na teoria dos Conjuntos Nebulosos ("Fuzzy Sets") tem se mostrado mais adequada para tratar imperfeições da informação do que a teoria das probabilidades (Interesssante notar que a teoria dos conjuntos nebulosos apareceu antes da lógica nebulosa, em um artigo que se tornou célebre de 1965 [19]). De forma mais objetiva e preliminar, podemos definir Lógica Nebulosa como sendo uma ferramenta capaz de capturar informações vagas, em geral descritas em uma linguagem natural e convertê-las para um formato numérico, de fácil manipulação pelos computadores de hoje em dia. Considere a seguinte afirmativa: Se o tempo de um investimento é longo e o sistema financeiro tem sido não muito estável, então a taxa de risco do investimento é muito alta. Os termos "longo", "não muito estável" e "muito alta" trazem consigo informações vagas.

\section{Características da Lógica Nebulosa}

- A Lógica Nebulosa está baseada em palavras e não em números, ou seja, os valores verdades são expressos lingüisticamente. Por exemplo: quente, muito frio, verdade, longe, perto, rápido, vagaroso, médio, etc.

- Possui vários modificadores de predicado como por exemplo: muito, mais ou menos, pouco, bastante, médio, etc.

- Possui também um amplo conjunto de quantificadores, como por exemplo : poucos, vários, em torno de, usualmente.

- Faz uso das probabilidades lingüísticas, como por exemplo: provável, improvável, que são interpretados como números nebulosos e manipulados pela sua aritmética.

- Manuseia todos os valores entre 0 e 1 , tomando estes, como um limite apenas [17].

\section{Vantagens}

- Requer poucas regras, valores e decisões;

- Mais variáveis observáveis podem ser valoradas;

- O uso de variáveis lingüísticas nos deixa mais perto do pensamento humano;

- Simplifica a solução de problemas;

- Proporciona um rápido protótipo dos sistemas;

- Simplifica a aquisição da base do conhecimento.

\subsubsection{Estudo de caso}


As variáveis impostas nessa pesquisa foram escolhidas a partir de uma avaliação de uma equipe médica conceituada no mercado de saúde. Foi escolhida a estratégia de entrevista aberta, onde o foco sempre foi voltado para Medicina Preventiva do indivíduo que no caso é o nosso cliente.

Sendo assim, as variáveis escolhidas foram as seguintes:

- Índice de Massa Corporal;

- Pressão arterial;

- Dígito indicativo de sedentarismo;

- Hábitos alimentares (gordura saturada, açúcar, sal, entre outros que se correlacionam com problemas de saúde);

- Tabagismo;

- Alcoolismo;

- Dependência por drogas;

- Nível de gravidade no primeiro atendimento médico;

- Histórico familiar (principais doenças correlacionadas a essa variável)

\section{8 . Análise de resultados:}

A partir do uso da ferramenta Matlab e utilizando a Fuzzy Logic Toolbox [8], foi definido o seguinte sistema de inferência:

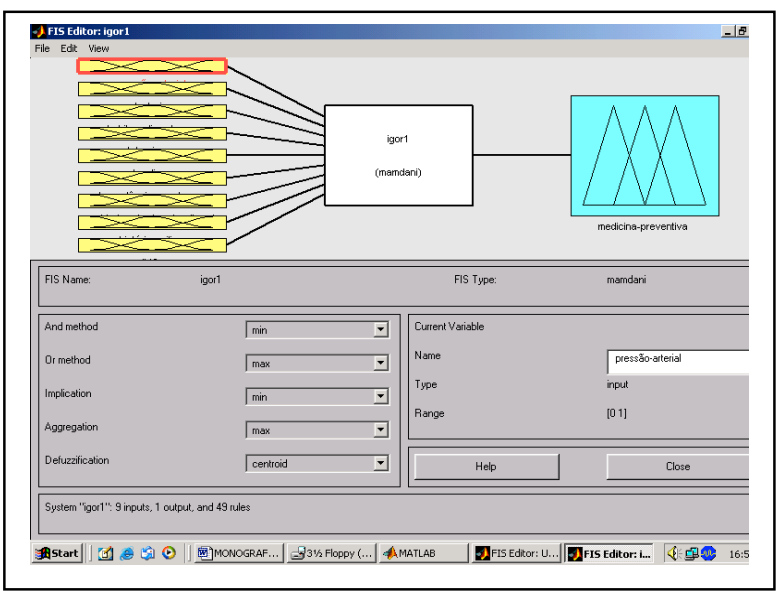

Figura 1- Sistema de Inferência do Fuzzy Toolbox do Matlab

O primeiro passo foi à definição das saídas deste sistema onde foi considerada apenas a opção de Medicina Preventiva, subdividida em Primária, Secundária e Terciária.

O objetivo desse sistema é desenhar o risco do cliente durante uma entrevista qualificada. Devido à automação desse processo surge o benefício da resposta em tempo real e não sendo necessário ser aplicada por um médico. Isso caracteriza um custo de oportunidade para empresa, já que esse trabalho poderá ser realizado por enfermeiras,m gerando assim um menor custo.

Todas as variáveis deste modelo foram normalizadas a partir das informações obtidas nos textos provenientes das entrevistas com médicos de diferentes áreas.

A primeira variável estudada foi IMC, que apresentou os aspectos abaixo, subdivididos de acordo com a tabela utilizada na medicina. Essa tabela nos mostra os valores que representam aspectos físicos do indivíduo e a partir dela evoluiu esta apresentação.

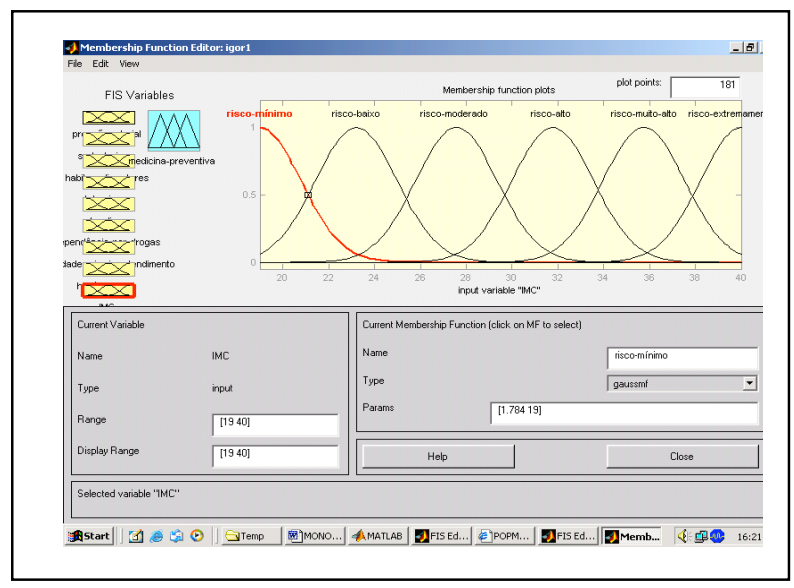

Figura 2 - Exemplo das variáveis de entrada

A segunda variável foi a pressão arterial que foi escolhida por ser um ótimo indicador de saúde do indivíduo, além de ser de rápida aferição. Ela se apresentou da seguinte maneira:

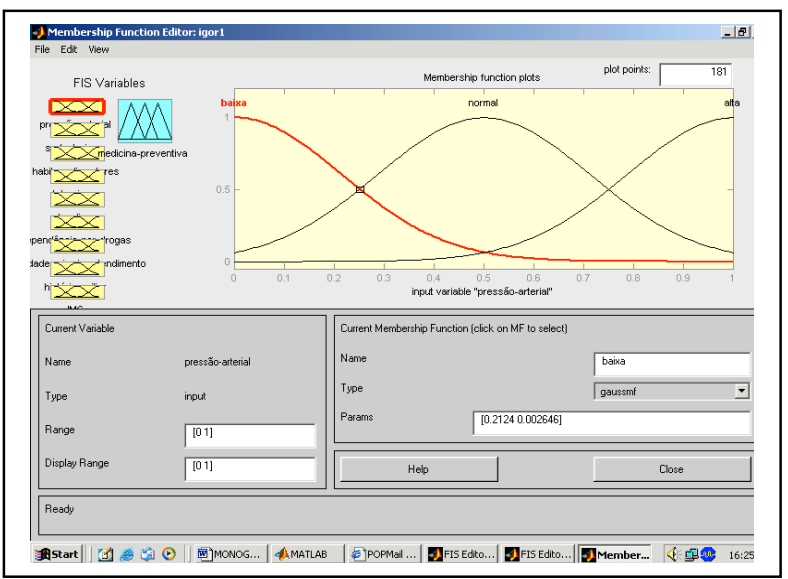

Figura 3 - Representação da variável pressão arterial

A terceira variável estudada foi o sedentarismo que está normalizada a partir da cultura do indivíduo. Isso por que há pessoas que não praticam esporte, mas possuem uma rotina de vida que para aspectos físicos é importante, exemplo: pessoas que caminham muito até chegar ao trabalho. 


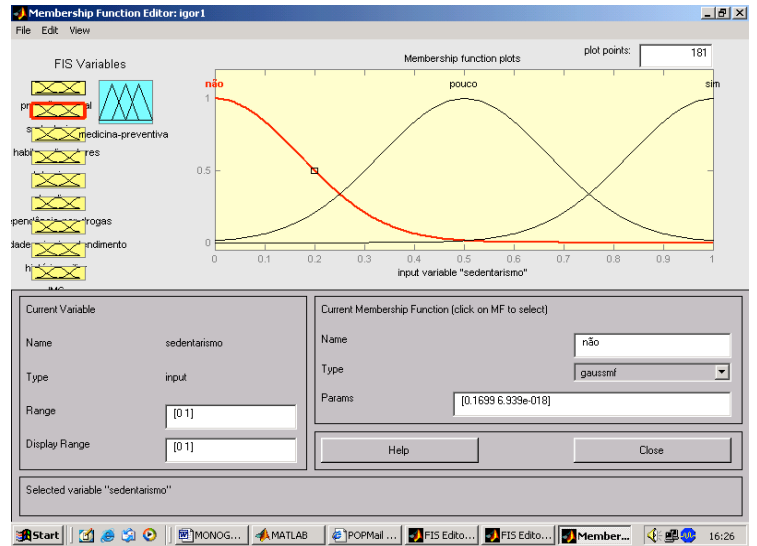

Figura 4 - Representação da variável sedentarimo

A quarta variável, hábitos alimentares foi normalizada a partir de uma analise cruzada com alimentos que são consumidos em excesso e são diretamente relacionadas com patologias , por exemplo: gordura - colesterol, sal - pressão alta e diabetes - açúcar. Apresentou-se da seguinte maneira:

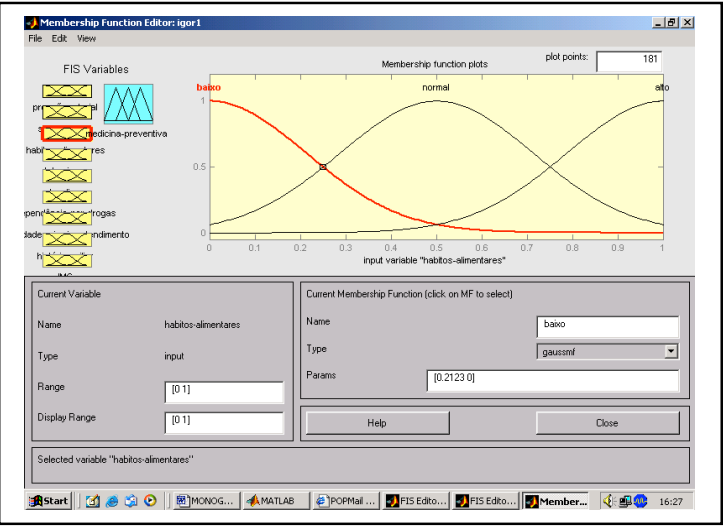

Figura 5 - Representação da variável hábitos alimentares

A quinta variável, o tabagismo foi normalizada a partir da quantidade de fumo consumido pelo cliente, estudando até mesmo se o indivíduo é um fumante passivo. Apresentou-se da seguinte maneira:

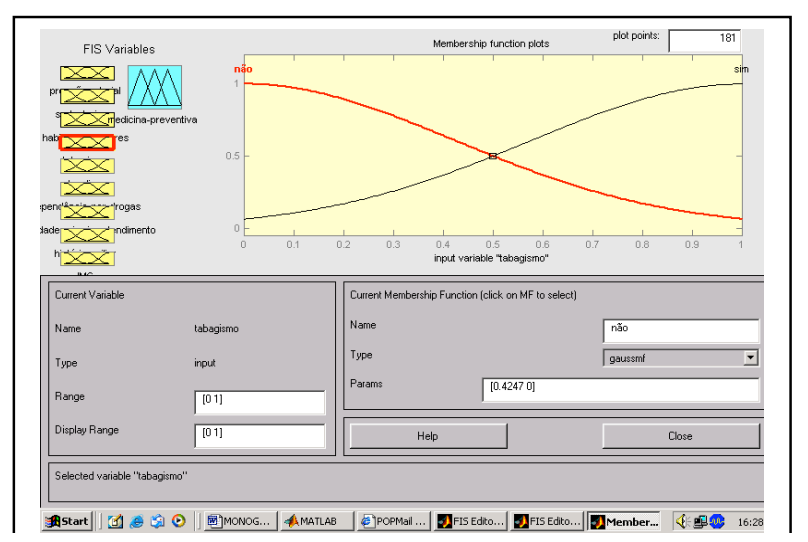

Figura 6 - Representação da variável tabagismo A sexta variável, o alcoolismo foi normalizado, assim como foi o tabagismo. Apresentou-se da seguinte maneira:

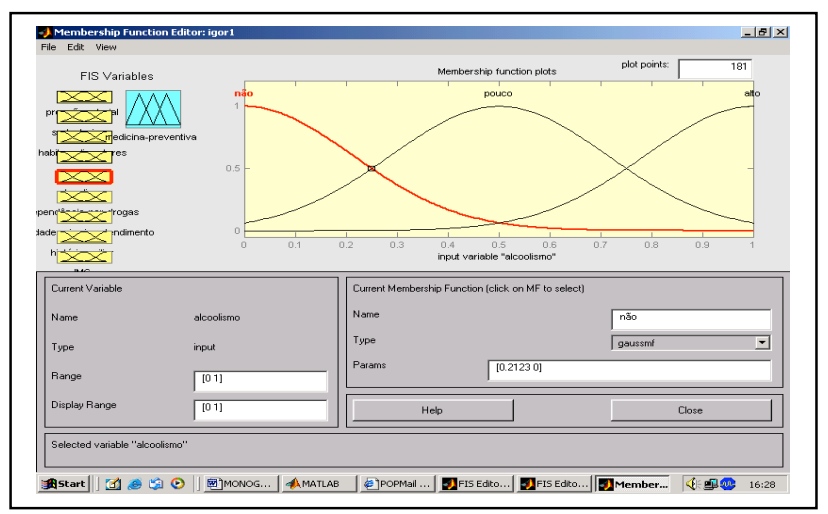

Figura 7 - Representação da variável alcoolismo

A sétima variável, dependência de drogas, também foi normalizada como o tabagismo e se apresentou da seguinte forma:

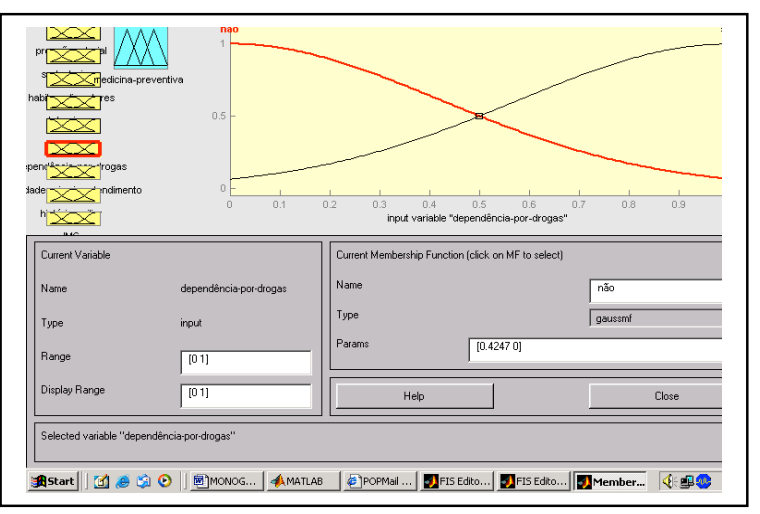

Figura 8 - Representação da dependência de drogas

A oitava variável, gravidade do primeiro atendimento , foi normalizada a partir do risco, ou seja: baixo, pequeno, médio, alto e muito alto. Essa variável é estudada quando há registro de um evento pós contrato, ou seja, não necessariamente ela será avaliada no momento da entrevista o que caracteriza a grande importância da mesma pelo fato de existir com isso um estudo contínuo do risco

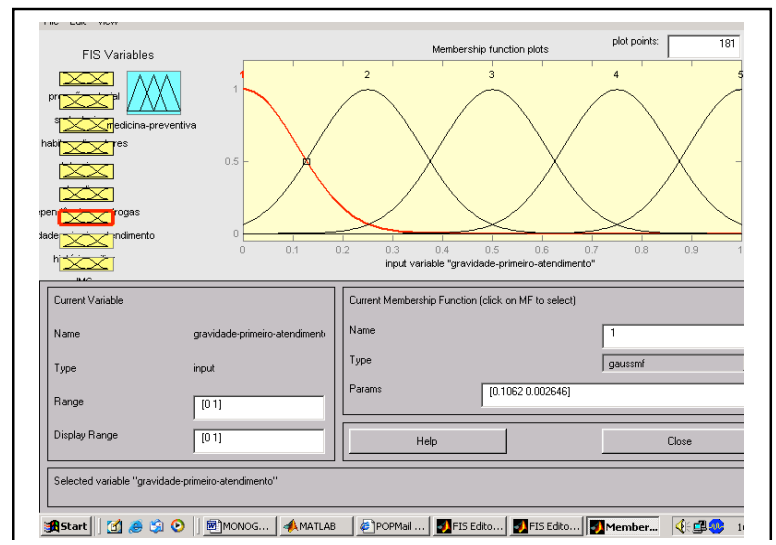


Figura 9 - Representação da gravidade do primeiro atendimento

E a última variável, histórico familiar foi normalizado tendo como premissa acontecimentos passados com os familiares do cliente onde é estudado eventos médicos ocorridos com pais e avós, mas indiretamente, através de questionário ou acesso a informações dos hospitais no qual tenham ido pela última vez.

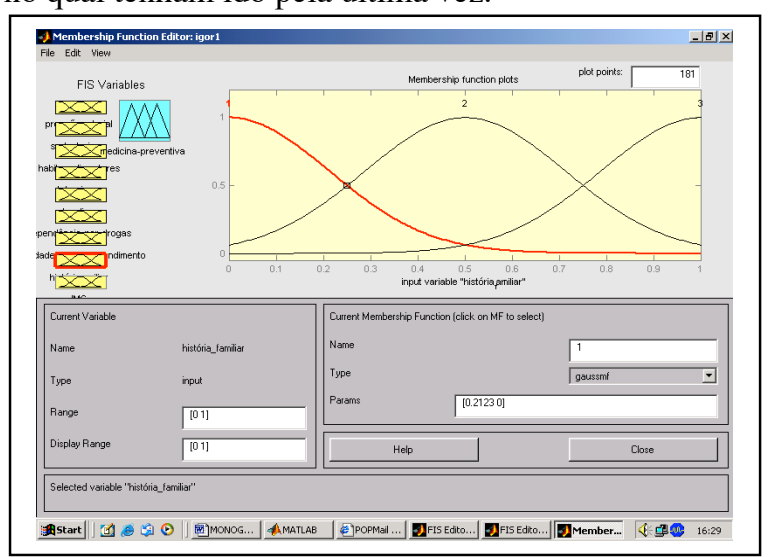

Figura 10 - Representação do histórico familiar

Todas as regras foram devidamente analisadas tendo como base as informações passadas pelos médicos consultados. A combinação das variáveis gerou as saídas do modelo. Foram criadas apenas 49 regras. Outras combinações poderão surgir, mas será realizado em trabalhos futuros. A apresentação no Matlab segue abaixo:

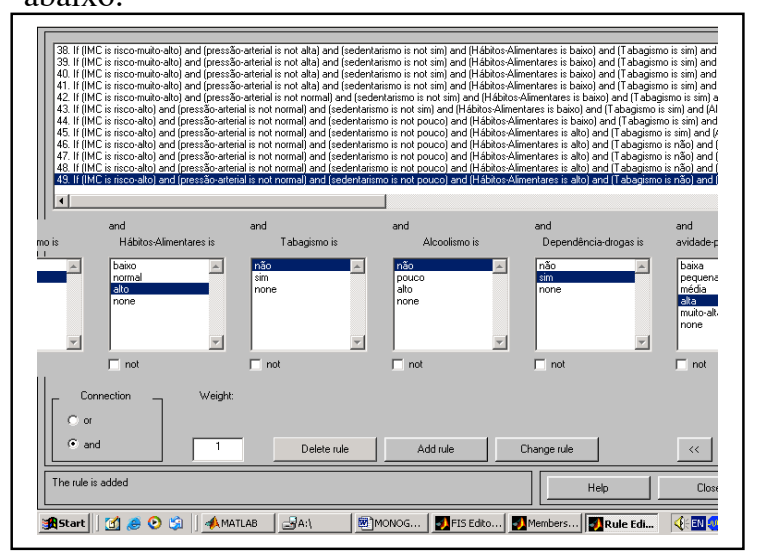

Figura 11 - Representação da base de regras utilizada

Chegando ao seguinte resultado:

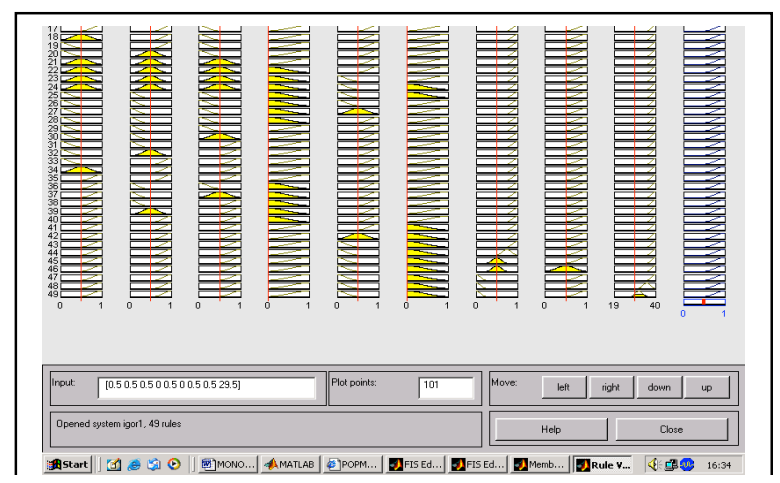

Figura 12 - Sistema de Inferência Nebuloso

O campo Input apresentado acima, representa os valores a serem inseridos pós entrevista. Ou seja, cada resposta será representada por um número o qual irá gerar diferentes resultados (output). O exemplo acima caracteriza uma saída para Medicina Preventiva Secundária onde o cliente em estudo apresentou: IMC com risco moderado, Pressão arterial normal, um pouco sedentário, hábitos alimentares normais, não fuma, bebe um pouco, não usa drogas, possui um risco médio para Gravidade no primeiro atendimento e por último possui um risco médio no Histórico familiar. A equipe médica avaliou este perfil de cliente como secundário, principalmente pelas duas últimas variáveis sendo o histórico familiar considerado com $60 \%$ de chance de reincidência.

\section{Metodologia}

\subsection{Tipo de pesquisa}

Observação simples e pesquisa de campo aberta.

\subsection{Coleta de dados}

Todas as variáveis foram selecionadas a partir de uma pesquisa com o tema: Quais variáveis identificam-se melhor na a avaliação de um paciente. Com foco na necessidade básica de prover uma melhor qualidade de vida e prover uma vida saudável, com o objetivo de minimizar os riscos de futuros problemas de saúde.

\subsection{Limitações do método}

Infelizmente, não será possível para o presente trabalho o estudo de todas as varáveis necessárias para uma melhor definição dos problemas de saúde de um cliente e seus respectivos protocolos, devido o grande quantidade de especificações técnicas.

\section{Conclusão}

A conclusão a que se chega neste trabalho é a da viabilidade de geração de novos canais de distribuição dos custos médicos através de técnicas administrativas que podem diminuir o custo das operadoras em pesquisa, evitando o retrabalho, já que manipular a informação de um paciente com problemas só depois que ocorrem os eventos não gera eficiência. 
A capacidade de se medir o risco dos novos clientes se demonstrou muito eficiente e a sua automação permitiu uma maior velocidade no tempo de ação o que justifica esse projeto.

Além de agregar valores para a área médica, há a potencializarão das vendas e melhoramento do marketing da empresa, já que o trabalho desta forma é visto como socialmente responsável, desejando que seu cliente viva mais e feliz.

\section{Bibliografia}

[0] A Associação Internacional de Sistemas Nebulosos (The International Fuzzy Systems Association (IFSA) é uma organização dedicada a suporte, desenvolvimento e promoção da Lógica Nebulosa). Dietary Guidelines, National Institute of Health, 1995. Disponível em: http://www.austinlinks.com/Fuzzy/

[1] Agência Nacional de Saúde Suplementar. Câmara de Saúde Suplementar - Atas de 31 reuniões. Rio de Janeiro: ANS, 2003. Disponível em: www.ans.gov.br

[2] ANDRADE, A .G.; NICASTRI, S.; TONGUE, E. eds. Drogas: Atualização em Prevenção e Tratamento. São Paulo, Lemos, 1993, pp.:153-64.

[3] Banco Mundial. Relatório sobre o desenvolvimento mundial 1993 - Investindo em Saúde. Rio de Janeiro: Fundação Getúlio Vargas

[4] Indicadores, ABRAMGE. Disponível em: www.abramge.com.br

[5] Folha de São Paulo. Disponível em: http://www1.folha.uol.com.br/fsp/

[6] JURUENA, M. Função Regulatória. Revista Dialogo Jurídico, 11, 2002. Disponível em: http://www.direitopúblico.com.br.

[7] MATHWORKS - Matlab 6.5

[8] McARDLEe, W.D.; KATCH, F.I.; KATCH, V. Fisiologia do Exercício, Energia, Nutrição e Desempenho Humano. - $3^{\mathrm{a}}$ edição. Guanabara Koogan, 1998.

[9] MENEZELlo, M. D'A. C. Agências Reguladoras e o Direito Brasileiro. São Paulo: Atlas, 2002.

[10] Ministério da Saúde. Secretaria Nacional de Assistência à Saúde. Instituto Nacional do Câncer. Disponível em: http://portal.saude.gov.br/saude/

[11] MINSKY, Marvin; PAPERT, Sigmour. Perceptrons, 1969.

[12] NETO, Turibio Leite de Barros - Exercício, Saúde e Desempenho Físico.- $1^{\mathrm{a}}$ edição. Editora Atheneu, São Paulo, Brasil, 1997.

[13] Princípio de Integridade a Saúde. Disponível em: www.planalto.gov.br/ccivil_03/Constituicao/Constitu i\%C3\%A7ao.htm

[14] Rand Corporation. Disponível em: www.rand.org
[15] Redes neurais. Disponível em: http://neuron.princeton.edu/ john/

[16] SHAW, Ian S.; SIMÕES, Marcelo Godoy. Controle e modelagem fuzzy. São Paulo: Edgard Blücher, 1999.

[17] TOJAL, S. B. De B. Controle Judicial da Atividade Normativa das Agências

[18] WAYT, Gibbs, "Gaining on Fat," Scientific American, Issue \#8 1996.

[19] ZADEH, L. "Fuzzy Sets", Information and Control, 8, 1, p.338-353, Jan. 1965. 